\title{
Acute and late vaginal toxicity after adjuvant high-dose-rate vaginal brachytherapy in patients with intermediate risk endometrial cancer: is local therapy with hyaluronic acid of clinical benefit?
}

\author{
Concetta Laliscia, MD', Durim Delishaj, MD', Maria Grazia Fabrini, MD', Alessandra Gonnelli, MD', Riccardo Morganti, MD², \\ Franco Perrone, MD³ , Roberta Tana, MD4, Fabiola Paiar, PhD!, Angiolo Gadducci, PhD ${ }^{4}$ \\ 'Department of Translational Medicine, Division of Radiation Oncology, ${ }^{2}$ Department of Experimental and Clinical Medicine, \\ Section of Statistics, ${ }^{3}$ Department of Translational Medicine, U. O Fisica Sanitaria, ${ }^{4}$ Department of Experimental and Clinical Medicine, \\ Division of Gynecology and Obstetrics, University of Pisa, Pisa, Italy
}

\begin{abstract}
Purpose: The aim of the present study was to evaluate the effectiveness of hyaluronic acid (HA) in the prevention of acute and late vaginal toxicities after high-dose-rate (HDR) vaginal brachytherapy (BT).

Material and methods: Between January 2011 and January 2015, we retrospectively analyzed 126 patients with endometrial cancer who underwent extrafascial hysterectomy with or without lymphadenectomy and adjuvant HDR-vaginal BT +/- adjuvant chemotherapy. The total dose prescription was $21 \mathrm{~Gy}$ in 3 fractions (one fraction for week). Vaginal ovules containing $5 \mathrm{mg}$ of HA were given for whole duration of vaginal BT and for the two following weeks. Acute and late toxicities were evaluated according to CTCAE vs 4.02.

Results: According to the revised FIGO 2009 classification, most tumors were in stage IA (30.9\%) and in stage IB (57.9\%). Thirty-three patients (26.2\%) received adjuvant chemotherapy before vaginal BT. Five-year disease-free survival (DFS) and five-year overall survival (OS) were $88 \%$ and $93 \%$, respectively. The most common grade 1-2 acute toxicities were vaginal inflammation (18 patients, $14.3 \%$ ) and dyspareunia (7 patients, $5.5 \%)$. Two patients $(1.6 \%)$ had more than one toxicity. Late toxicity occurred in 20 patients (15.9\%). Grade 1-2 late toxicities were fibrosis (14 patients, $11.1 \%)$ and telangiectasias (7 patients, $5.5 \%)$. Six patients (4.8\%) had more than one late toxicity. No grade 3 or higher acute or late toxicities were observed.

Conclusions: These results appear to suggest that the local therapy with HA is of clinical benefit for intermediate risk endometrial cancer patients who receive adjuvant HDR-vaginal BT after surgery. A randomized trial comparing HA treatment vs. no local treatment in this clinical setting is warranted to further evaluate the efficacy of HA in preventing vaginal BT-related vaginal toxicity.
\end{abstract}

Key words: endometrial cancer, HDR vaginal brachytherapy, hyaluronic acid.

\section{Purpose}

The standard treatment of endometrial cancer consists of laparotomy, peritoneal washing, total extra-fascial hysterectomy, and bilateral salpingo-oophorectomy with or without pelvic \pm aortic lymphadenectomy $[1,2,3]$. In the last years, laparoscopic and robotic-assisted laparoscopic hysterectomy have increasingly used with very satisfactory results, and nowadays, minimally invasive surgery may be considered as a treatment of choice, especially in morbidly obese women [4,5].

A recent meta-analysis has shown that adjuvant pelvic external-beam radiotherapy (EBRT) reduces the risk of loco-regional recurrence with an odds ratio (OR) of $0.36(95 \%$ confidence interval $[\mathrm{CI}]=0.25-0.52)$, without any improvement in terms of overall survival (OS) ( $\mathrm{HR}=0.99,95 \%$ CI: 0.82-1.20), endometrial cancer-specific survival $(\mathrm{HR}=0.96,95 \% \mathrm{CI}: 0.72-1.28)$, or distant recurrence rates (risk ratio $[R R]=1.04,95 \%$ CI: 0.80-1.35) [6]. In a previous meta-analysis, Johnson and Cornes [7] have reported that adjuvant EBRT is detrimental (OR for OS $=0.71 ; 95 \%$ CI: 0.52-0.96) in low-risk endometrial cancer (defined as stage IA-IB, grade [G] 1-2, FIGO 1988), it does not affect OS (OR $=0.97 ; 95 \%$ CI: 0.69-1.35) in intermediate-risk disease (defined as either stage IC, G1-2 or stage IB G3, FIGO 1988), and it gives a significant OS benefit

Address for correspondence: Concetta Laliscia, MD, Department of Translational Medicine, Division of Radia- Received: 04.08 .2016 tion Oncology, University of Pisa, Azienda Ospedaliero Universitaria Pisana, Department of Radiotherapy, Accepted: 17.11 .2016 Via Roma 67, Pisa, Italy, phone: +39 050 993456, fax: +39 050 992960, e-mail: c.laliscia@ao-pisa.toscana.it Published: 30.12 .2016 
$(\mathrm{OR}=1.76 ; 95 \% \mathrm{CI}: 1.07-2.89)$ in high-risk disease (defined as stage IC G3, FIGO 1988).

Vaginal brachytherapy (BT) alone has been used as adjuvant treatment, especially in stage IB G1-2 disease, stage IA G3 disease, and stage IC G1-2 disease, with satisfactory results in terms of local control and with low toxicity $[8,9,10]$.

The Post-Operative Radiation Therapy in Endometrial Cancer (PORTEC) 2 trial randomly allocated 427 patients with intermediate-high risk endometrial cancer to receive either adjuvant vaginal BT (21 Gy with high-doserate [HDR] or $30 \mathrm{~Gy}$ with low-dose-rate [LDR]) or adjuvant pelvic EBRT (46 Gy) [11,12]. The two arms had similar 3-year vaginal recurrence rates $(0.9 \%$ for vaginal BT vs. $2 \%$ for EBRT) and 3-year OS rates (90.4\% vs. $90.8 \%)$, whereas patients enrolled into vaginal BT arm experienced better social functioning, less bowel toxicity, and better quality of life (QoL). High-dose-rate-vaginal BT can be considered as an adjuvant radiotherapy of choice in intermediate risk patients after surgical staging including pelvic \pm aortic lymphadenectomy $[11,12,13,14,15,16]$. However, acute and long-term side effects, such as thinning and increasing fragility of vaginal walls, atrophy, adhesions, fibrosis, and stenosis, have been reported also after HDR-vaginal BT, with occasional impairment of sexual life $[8,9,10,11,12,13,14,15,17,18,19,20,21,22,23]$.

Hyaluronic acid (HA) is a polysaccharide belonging to the class of glycosaminoglycans, involved in the processes of tissue repair and regeneration [24]. It is a fundamental component of extracellular matrix, which contributes to an optimal performance of the repair processes by providing the suitable degree of hydration, which facilitates the cell migration.

The aim of the present study was to evaluate the effectiveness of topical therapy with hyaluronic acid in order to prevent acute and late vaginal toxicities in patients with endometrial cancer who underwent primary surgery and adjuvant HDR-vaginal BT with or without adjuvant chemotherapy.

\section{Material and methods}

\section{Patients and treatment characteristics}

The present investigation retrospectively analyzed 126 patients with early stage endometrial cancer who received adjuvant HDR-vaginal BT after extra-fascial hysterectomy and bilateral salpingo-oophorectomy, with or without pelvic + aortic lymphadenectomy, between January 2011 and January 2015. The patients were retrospectively staged according to the revised International Federation of Gynecology and Obstetrics (FIGO) classification 2009 [25]. Histological classification and grading were performed as per the American Joint Committee on Cancer (AJCC) criteria [26]. The architectural grade of endometrioid carcinomas was defined as follows: G1: $<5 \%$ of non-squamous or non-morular solid growth pattern; G2: $6-50 \%$ of non-squamous or non-morular solid growth pattern; G3: $>50 \%$ of non-squamous or non-morular solid growth pattern. Notable nuclear atypia, inappropriate for the architectural grade, raised the grade of G1 or G2 tumors by one.
Adjuvant treatment planning was established for each individual patient following comprehensive discussion between gynecologist oncologists and radiotherapists within a multidisciplinary team.

All patients underwent computerized tomography (CT) simulation (GE LightSpeed RT, GE Healthcare, MediPhysics Inc., Arlington Heights, IL, USA) with CT simulator $2.5 \mathrm{~mm}$ slice thicknesses in supine position for a better delineation of the clinical target volume (CTV), and dose to the vaginal mucosa, normal tissue, and structures. Prior to simulation, patients underwent vaginal exploration and introduction of a properly sized vaginal single channel cylinder applicator. The diameter of vaginal cylinder (range: $2-3.5 \mathrm{~cm}$ ) was selected considering vaginal size and extension, in order to optimize dose distribution and to decrease side effects. According to the American Brachytherapy Society consensus guidelines, target delineation was contoured by a radiation oncologist. For adjuvant treatment of the vaginal cuff after hysterectomy, the proximal $(5 \mathrm{~cm})$ of the vagina was treated. The dose prescription point was individually selected and the dose has been reported at the vaginal surface and in $0.5 \mathrm{~cm}$ depth [27].

Following the applicator placement by a radiation oncologist, we used a personalized immobilizing device to minimize movement between planning and treatment delivery. The total dose prescription was $21 \mathrm{~Gy}$ in three fractions of $7 \mathrm{~Gy}$ (one fraction for week) calculated in 0.5 $\mathrm{cm}$ depth. Recalculated to $2 \mathrm{~Gy}$ equivalent dose, the total dose was 42 Gy at $5 \mathrm{~mm}(\alpha / \beta=3)$ [28].

Vaginal ovules containing $5 \mathrm{mg}$ of HA (with aloe, centella asiatica, and calendula extracts) were applied for the entire duration of vaginal BT and for the following two weeks thereafter. This topical treatment was administered once a day in asymptomatic patients and twice daily in those with vaginal symptoms (inflammation with or without discharge).

Vaginal mucosal toxicity was evaluated during gynecological examination one month after vaginal BT completion and then every two months for the first year, every three months for the second year, and every six months thereafter. Vaginal toxicity was assessed based on both the symptoms reported by the patient and mucosal changes noted during gynecological examination. If necessary, a colposcopy of the vagina and vaginal dome was performed. Any symptoms that persisted or developed six months following vaginal BT completion were classified as late toxicity.

Acute and late toxicity was graded according to the Common Terminology Criteria for Adverse Events (CTCAE), version 4.02 [29]. The use of vaginal dilators was not prescribed.

All patients were periodically followed-up, until they died or up to July 2016. The median follow-up of survivors was 29 months (range: 3-59 months).

\section{Statistical analysis}

Qualitative data were analyzed by $x^{2}$ test or Fisher exact test when appropriate.

Disease-free survival (DFS) and OS were calculated from the end of vaginal BT. Survival probability was 
Table 1. Patients and treatment characteristics

\begin{tabular}{|c|c|c|}
\hline Characteristics & No & $\%$ \\
\hline All patients & 126 & \\
\hline \multicolumn{3}{|l|}{ Age (years) } \\
\hline Median (range: 27-90) & 67 & \\
\hline \multicolumn{3}{|l|}{ Surgery } \\
\hline Hystero-annessectomy & 126 & \\
\hline \multicolumn{3}{|l|}{ Histological types } \\
\hline Type I & 100 & 79.4 \\
\hline Type II & 26 & 20.6 \\
\hline \multicolumn{3}{|l|}{ Grading } \\
\hline G1 & 32 & 25.4 \\
\hline G2 & 71 & 56.3 \\
\hline G3 & 23 & 18.3 \\
\hline \multicolumn{3}{|l|}{ FIGO classification } \\
\hline IA & 39 & 30.9 \\
\hline $\mathrm{IB}$ & 73 & 57.9 \\
\hline ॥ & 11 & 8.8 \\
\hline III & 3 & 2.4 \\
\hline \multicolumn{3}{|l|}{ Radiotherapy } \\
\hline HDR-BT 21 Gy/3 fr (1 fraction/week) & 126 & \\
\hline \multicolumn{3}{|l|}{ Adjuvant chemotherapy } \\
\hline Yes & 33 & 26.2 \\
\hline No & 93 & 73.8 \\
\hline \multicolumn{3}{|l|}{ Follow-up (months) } \\
\hline Median (range: 3-59) & 29 & \\
\hline
\end{tabular}

FIGO - Federation Internationale de Gynecologie et d'Obstetrique, HDR-BT high-dose-rate brachytherapy, fr - fractions

estimated using the Kaplan-Meier method. Differences were considered significant at $p<0.05$. Analyses were performed using the SPSS v. 23 software (IBM, Armonk, NY, USA).

\section{Results}

Patient characteristics are shown in Table 1 . The median age of patients was 67 and histologically $79.4 \%$ of tumors were type 1 endometrioid carcinomas. Tumor grade was G2 and G3 in $56.3 \%$ and $18.3 \%$ of cases, respectively. According to the revised FIGO 2009 classification, most tumors were in stage IA $(30.9 \%)$ or in stage IB $(57.9 \%)$. The three women with stage FIGO III disease, who received vaginal $\mathrm{BT}$ as an exclusive adjuvant treatment were older than 85 and had severe concomitant comorbidities.

Thirty-three patients $(26.2 \%)$ received adjuvant chemotherapy consisting of paclitaxel $175 \mathrm{mg} / \mathrm{m}^{2}$ plus car- boplatin area under the curve (AUC) 5 (every three weeks for 4-6 cycles) in 28 women and single-agent carboplatin AUC 5 (every three weeks for 4-6 cycles) in five women. Twelve $(9.5 \%)$ patients recurred after a median interval of 35 months. The site of recurrence was vaginal in five patients $(3.9 \%)$, pelvic lymph nodal in three $(2.4 \%)$, both vaginal and pelvic lymph nodal in two $(1.6 \%)$, and distant in two patients $(1.6 \%)$ (peritoneal carcinosis in one case and bone metastases in the other).

Five-year DFS and five-year OS were $88 \%$ and $93 \%$, respectively (Figure 1 and 2). As shown in Table 2, overall acute toxicity was observed in 24 patients (19.0\%). The most common acute toxicities were vaginal inflammation $(14.3 \%)$ and dyspareunia (5.5\%). Two patients (1.6\%) had more than one toxicity. Late toxicity occurred in 20 patients $(15.9 \%)$. The most common late toxicities were fibrosis $(11.1 \%)$ and telangiectasias $(5.5 \%)$. Six patients $(4.8 \%)$ had more than one late toxicity. No grade 3 acute or late toxicities were observed. Seventeen women $(13.5 \%)$ had both acute and late toxicities. No statistical differences in terms of acute or late vaginal toxicities were observed between patients who underwent adjuvant chemotherapy and those who did not ( $p$ value $=0.6$, Fisher's test $)$.

\section{Discussion}

High-dose-rate-vaginal BT is often preferred as an adjuvant therapy following surgery in patients with intermediate-risk endometrial cancer $[11,12,13,14,15,16,17,18]$. However, a lack of data exists concerning acute and late toxicities after HDR-vaginal BT as exclusive postoperative radiotherapy.

MacLeod et al. [13] used HDR-vaginal BT only (34 Gy in four fractions prescribed to the vaginal mucosa in a two-week period) in 143 patients who underwent surgery for endometrial cancer. Five-year DFS and five-year OS were $100 \%$ and $88 \%$ for stage IA (FIGO 1988), $98 \%$ and $94 \%$ for stage $\mathrm{IB}, 100 \%$ and $86 \%$ for stage $\mathrm{IC}$, and $92 \%$ and $92 \%$ for stage IIA, respectively. The overall vaginal recurrence rate was $1.4 \%$ and the overall late-toxicity rate was low with no grade $\geq 3$ toxicities. Thirty-three patients (23.1\%) had at least one late toxicity. The most common toxicities were vaginal toxicity (grade 1 in 20 [13.9\%] patients and grade $2[1.4 \%]$ in two patients), and cystitis (8 [5.6\%] patients). In a prospective study by Eltabbakh $e t$ al. [14], 303 patients with stage IB (FIGO 1988), G1-2 endometrial carcinoma underwent postoperative LDR vaginal BT (30 Gy to a $0.5 \mathrm{~cm}$ depth). Six patients died of recurrent disease, but there were no vaginal recurrences. Fiveand 10 -year DFS rates were $98.9 \%$ and $97.8 \%$, respectively. Major vaginal BT-related complications occurred in seven $(2.1 \%)$ patients and consisted of severe vaginal stenosis in four $(1.2 \%)$, cystitis in three $(0.9 \%)$, colitis in one $(0.3 \%)$, and recto-vaginal fistula in one patient $(0.3 \%)$. One patient had both cystitis and vaginal stenosis, and another had both colitis and vaginal stenosis.

Sorbe et al. [15] randomly allocated 290 patients with stage IA-IB (1988), G1-G2 endometrial carcinoma to received adjuvant HDR-vaginal BT at a dose of either $15 \mathrm{~Gy}$ or $30 \mathrm{~Gy}$, given in six fractions over an eight-day period; the dose per fraction was randomly assigned to 
Table 2. Acute and late vaginal toxicity after adjuvant high dose rate (HDR) vaginal brachytherapy (21 Gy delivered in three fractions)

\begin{tabular}{|c|c|c|c|c|}
\hline \multirow[t]{2}{*}{ Grading } & \multicolumn{4}{|c|}{ Acute toxicity } \\
\hline & G1 & G2 & G3 & Any G \\
\hline Vaginal inflammation & 14 & 4 & 0 & $18(14.3 \%)$ \\
\hline Dyspareunia & 6 & 1 & 0 & $7(5.5 \%)$ \\
\hline Hemorrhage & 0 & 1 & 0 & $1(0.8 \%)$ \\
\hline All & $20(15.8 \%)$ & $6(4.8 \%)$ & $0(0 \%)$ & $26(20.6 \%)$ \\
\hline \multirow[t]{2}{*}{ Grading } & \multicolumn{4}{|c|}{ Late Toxicity } \\
\hline & G1 & $\mathrm{G} 2$ & G3 & Any G \\
\hline Dryness & 4 & 2 & 0 & $6(4.8 \%)$ \\
\hline Telangiectasias & 6 & 1 & 0 & $7(5.5 \%)$ \\
\hline Fibrosis & 13 & 1 & 0 & $14(11.1 \%)$ \\
\hline Stenosis & 2 & 0 & 0 & $2(1.6 \%)$ \\
\hline All & 25 (19.9\%) & $4(3.1 \%)$ & 0 & $29(23.0 \%)$ \\
\hline
\end{tabular}

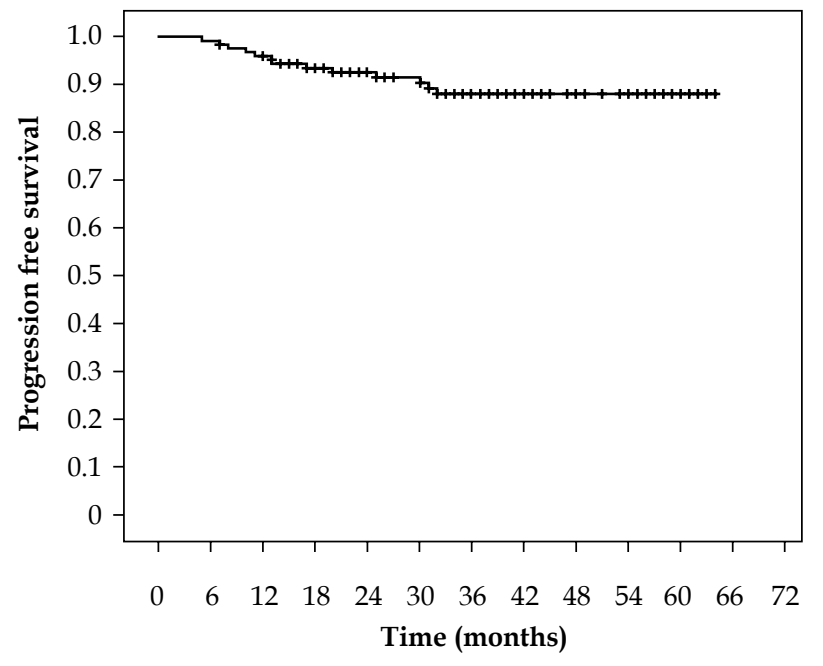

$\rightarrow$ Survival function $\quad+$ Censored

Fig. 1. Five years - progression free survival

2.5 Gy or 5.0 Gy. Recalculated to 2 Gy equivalent doses, the total doses compared were 16.5 Gy and 48.0 Gy at $5 \mathrm{~mm}$, and $30.4 \mathrm{~Gy}$ and $94.5 \mathrm{~Gy}$ at the surface of the vaginal applicator $(\alpha / \beta=3)$. The dose per fraction was specified at a depth of $5 \mathrm{~mm}$ from the surface of the vaginal cylinder using the HDR technique. The overall loco-regional recurrence rate was $1.4 \%$ and vaginal recurrence rate was $0.7 \%$, with no difference between the two arms. Mucosal atrophy and bleeding were more frequent in the higher dose group.

Chong and Hoskin [16] administered postoperative vaginal BT only (22 Gy, delivering a dose of 5.5 Gy per fraction at a depth of $5 \mathrm{~mm}$ from the applicator surface) to 173 patients with early stage endometrial carcinoma. There were 19 deaths, six (3.5\%) from disseminated tumor and $13(7.5 \%)$ from unrelated causes. All six patients

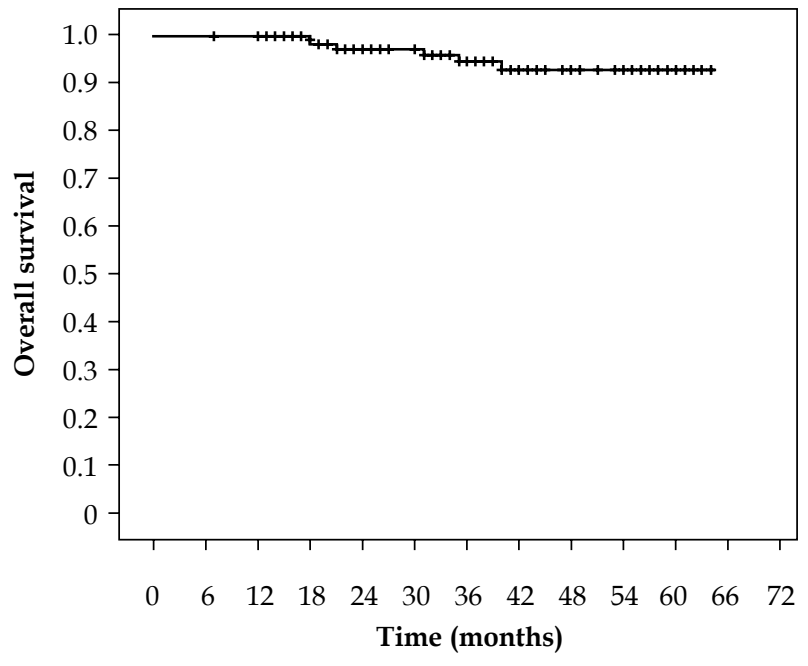

$\neg$ Survival function $\quad+$ Censored

Fig. 2. Five years - overall survival

who developed metastases had stage 1C (FIGO 1988) or G3 disease or clear cell histology. Late morbidity was rare, except for vaginal stenosis seen in $13 \%$ of cases. Alektiar et al. [17] retrospectively assessed 382 patients with stage IB-IIB endometrial cancer treated with surgery, followed by HDR-vaginal BT (21 Gy, given in three fractions at a two-week interval and the dose was prescribed to a depth of $0.5 \mathrm{~cm}$ from the vaginal surface). The five-year vaginal/pelvic control rate was $95 \%$ and on multivariate analysis, loco-regional failure rate correlated with age $\geq 60$ years old ( $R R=3,95 \%$ CI: $1-12), G 3$ (RR $=9$, 95\% CI: 2-35), and lymph-vascular space involvement invasion ( $\mathrm{RR}=4,95 \% \mathrm{CI}: 1-13)$. Grade 3 or higher late toxicities were observed in three cases $(0.8 \%)$, and consisted of vaginal necrosis, chronic cystitis and urethral stricture, respectively. Nout et al. [30] in the PORTEC-2 study re- 
ported the results of 427 high-intermediate risk endometrial cancer patients underwent at hysterectomy and bilateral salpingo/oophorectomy, and EBRT $(n=214$, 46 Gy 5 fractions for week) and vaginal BT ( $n=213,21$ Gy at $5 \mathrm{~mm}$ depth in 3 fractions of 7 Gy over two weeks).

Vaginal BT patients reported better social functioning $(p$ value $=0.005)$ and lower symptom scores for diarrhea, fecal leakage, need to stay close to a toilet, and limitation in daily activities due to bowel symptoms $(p=0.001)$ compared to EBRT. In this study, there was an increase of grade 1 and 2 mucosal atrophy from 6 months onwards (at 3 years 17\% after EBRT vs. 35\% after VBT). Nevertheless, there were no significant differences in sexual functioning and sexual symptoms between patients treated with EBRT or vaginal BT.

Some authors have reported that the local administration of HA appears to reduce vaginal atrophy and related symptoms in women treated with radiotherapy for gynecological cancers $[31,32,33,34]$. This local therapy may favor regeneration and epithelial proliferation, and improve vaginal trophism, elasticity and sufficient lubrication. In addition, HA can markedly reduce the sticking of the vaginal walls, thus preventing the formation of adhesions, vaginal obliteration, and stenosis.

Markowska et al. [31] administered HA-containing ovules to 37 women with endometrial cancer who had vaginal inflammatory/necrotic lesions, adhesions of vaginal walls, and complaints associated with coitus following the completion of vaginal BT. Vaginal ovules were applied once daily for 10 days, every second day for one month and in responsive cases, every third day for an additional month. A clinical improvement was observed after three months in 32 (86.5\%) women.

In a study by Cassaro et al. [33], local therapy with HA reduced the incidence of vaginal atrophy, dryness, adhesions, and stenosis, as well as dyspareunia in 45 patients who underwent EBRT with or without vaginal BT for different gynecological cancers. Dinicola et al. [34] randomly allocated 45 cervical cancer patients treated with surgery, adjuvant concomitant chemotherapy, and EBRT and BT to receive local therapy with HA twice daily for four months vs. no additional local therapy. For the first two months, the preventive treatment was given together with radiotherapy. A biopsy performed after BT revealed a significant improvement in inflammation, cell atypia, fibrosis, mucositis, and bleeding in the treated group. A biopsy performed two months later showed a further improvement in all radiotherapy-related side effects for the HA arm.

\section{Conclusions}

Based on the results of our study, the use of topical therapy with HA appears to have a clinical benefit for intermediate-risk endometrial cancer patients who receive adjuvant HDR-vaginal BT following surgery. Since vaginal toxicity may occur early, we suggest applying vaginal $\mathrm{HA}$ at the beginning and during vaginal BT, and to continue for at least two weeks after the end of radiotherapy. A randomized trial comparing HA treatment vs. no local treatment in this clinical setting is warranted to further evaluate the efficacy of HA in preventing vaginal BT-related vaginal toxicity.

\section{Disclosure}

Authors report no conflict of interest.

\section{References}

1. Boronow RC. Surgical staging of endometrial cancer: evolution, evaluation, and responsible challenge - a personal perspective. Gynecol Oncol 1997; 66: 179-189.

2. American College of Obstetricians and Gynecologists. ACOG practice bulletin, clinical management guidelines for obstetrician-gynecologists, number 65, August 2005: management of endometrial cancer. Obstet Gynecol 2005; 106: 413-425.

3. Colombo N, Creutzberg C, Amant F et al. ESMO-ESGOESTRO Endometrial Consensus Conference Working Group. ESMO-ESGO-ESTRO consensus conference on endometrial cancer: Diagnosis, treatment and follow-up. Radiother Oncol 2015; 117: 559-581.

4. Chan JK, Gardner AB, Taylor K et al. Robotic versus laparoscopic versus open surgery in morbidly obese endometrial cancer patients - a comparative analysis of total charges and complication rates. Gynecol Oncol 2015; 139: 300-305.

5. Rabinovich A. Minimally invasive surgery for endometrial cancer. Curr Opin Obstet Gynecol 2015; 27: 302-307.

6. Kong A, Johnson N, Kitchener HC et al. Adjuvant radiotherapy for stage I endometrial cancer: an updated Cochrane systematic review and meta-analysis. J Natl Cancer Inst 2012; 104: 1625-1634.

7. Johnson N, Cornes P. Survival and recurrent disease after postoperative radiotherapy for early endometrial cancer: systematic review and meta-analysis. BJOG 2007; 114: 1313-1320.

8. Alektiar KM, McKee A, Venkatraman E et al. Intravaginal high-dose-rate brachytherapy for Stage IB (FIGO Grade 1, 2) endometrial cancer. Int J Radiat Oncol Biol Phys 2002; 53: 707-713.

9. Rittenberg PV, Lotocki RJ, Heywood MS et al. High-risk surgical stage 1 endometrial cancer: outcomes with vault brachytherapy alone. Gynecol Oncol 2003; 89: 288-294.

10. Jolly S, Vargas C, Kumar $\mathrm{T}$ et al. Vaginal brachytherapy alone: an alternative to adjuvant whole pelvis radiation for early stage endometrial cancer. Gynecol Oncol 2005; 97: 887-892.

11. Nout RA, Putter H, Jürgenliemk-Schulz IM et al. Quality of life after pelvic radiotherapy or vaginal brachytherapy for endometrial cancer: first results of the randomized PORTEC-2 trial. J Clin Oncol 2009; 27: 3547-3556.

12. Nout R, Smit V, Putter H et al. Vaginal brachytherapy versus pelvic external beam radiotherapy for patients with endometrial cancer of high-intermediate risk (PORTEC-2): an open label, non-inferiority, randomized trial. Lancet 2010; 375: 816-823.

13. MacLeod C, Fowler A, Duval P et al. High-dose-rate brachytherapy alone post-hysterectomy for endometrial cancer. Int J Radiat Oncol Biol Phys 1998; 42: 1033-1039.

14. Eltabbakh GH, Piver MS, Hempling RE et al. Excellent long term survival and absence of vaginal recurrences in 332 patients with low risk stage I endometrial adenocarcinoma treated with hysterectomy and vaginal brachytherapy without formal staging lymph node sampling: report of a prospective trial. Int J Radiat Oncol Biol Phys 1997; 38: 373-380.

15. Sorbe B, Straumits A, Karlsso L. Intravaginal high-dose-rate brachytherapy for Stage I endometrial cancer: A randomized study of two dose-per-fraction levels. Int J Radiat Oncol Biol Phys 2005; 62: 1385-1389. 
16. Chong J, Hoskin PJ. Vaginal vault brachytherapy as sole postoperative treatment for low risk endometrial cancer. Brachytherapy 2008; 7: 195-199.

17. Alektiar KM, Venkatraman E, Chi DS et al. Intravaginal brachytherapy alone for intermediate-risk endometrial cancer. Int J Radiat Oncol Biol Phys 2005; 62: 111-117.

18. Gadducci A, Greco C. The evolving role of adjuvant therapy in endometrial cancer. Crit Rev Oncol Hematol 2011; 78: 79-91.

19. Eifel PJ, Levenback C, Wharton JT et al. Time course and incidence of late complications in patients treated with radiation therapy for FIGO Stage 1B carcinoma of the uterine cervix. Int J Radiat Oncol Biol Phys 1995; 32: 1289-1300.

20. Nunns D, Williamson K, Swaney L et al. The morbidity of surgery and adjuvant radiotherapy in the management of endometrial carcinoma. Int J Gynecological Cancer 2000; 10: 233-238.

21. Lin LL, Mutch DG, Rader JS et al. External radiotherapy versus vaginal brachytherapy for patients with intermediate risk endometrial cancer. Gynecol Oncol 2007; 106: 215-220.

22. Chang J, Hoskin PJ. Vaginal vault brachytherapy as sole postoperative treatment for low risk endometrial cancer. Brachytherapy 2008; 7: 195-199.

23. Sapienza LG, Aiza A, Gomes MJ et al. Bladder (ICRU) dose point does not predict urinary acute toxicity in adjuvant isolated vaginal vault high-dose-rate brachytherapy for intermediate-risk endometrial cancer. J Contemp Brachytherapy 2015; 7: 357-362.

24. Gerdin B, Hällgren R. Dynamic role of hyaluronan (HA4) in connective tissue activation and inflammation. J Intern Med 1997; 242: 49-55

25. Lewin SN. Revised FIGO staging system for endometrial cancer. Clin Obstet Gynecol 2011; 54: 215-218.

26. Edge SC, David R, Compton CC et al. AJCC Cancer Staging Manual. 7 ed. Springer Science and Business Media, New York 2010.

27. Small W Jr, Beriwal S, Demans DJ et al. American Brachytherapy Society consensus guidelines for adjuvant vaginal cuff brachytherapy after hysterectomy. Brachytherapy 2012; 11: 58-67.

28. Withers HR, Thames HD Jr, Peters LJ. A new isoeffect curve for change in dose per fraction. Radiother Oncol 1983; 1: 187-191.

29. CTCAE v. 4.0: NIH Publication No. 09-5410.

30. Nout RA, Putter H, Jürgenliemk-Schulz IM et al. Five-year quality of life of endometrial cancer patients treated in the randomized Post-Operative Radiation Therapy in Endometrial Cancer (PORTEC-2) trial and comparison with norm data. Eur J Cancer 2012; 48: 1638-1648.

31. Markowska J, Madry J, Markowska A. The effect of hyaluronic acid (Cicatridine) on healing and regeneration of the uterine cervix and vagina and vulvar dystrophy therapy. Eur J Gynaecol Oncol 2011; 32: 65-68.

32. Katz A. Interventions for sexuality after pelvic radiation therapy and gynecological cancer. Cancer J 2009; 75: 45-47.

33. Cassaro N, Bianca G, Meli MT. Vaginal ovules containing hyaluronic acid in the treatment of the side effects of radiotherapy and chemotherapy on the vagina. Giorn It Ost Gin 2009; 31.

34. Dinicola S, Pasta V, Costantino D et al. Hyaluronic acid and vitamins are effective in reducing vaginal atrophy in women receiving radiotherapy. Minerva Ginecol 2015; 67: 523-531. 